\title{
ON THE CONSTRUCTION OF UNIVERSALLY OPTIMAL BLOCK DESIGNS WITH NESTED ROWS AND COLUMNS
}

\author{
HUNG LIN FU AND HSIAO TIEN PAO
}

\begin{abstract}
This paper presents a simple method for constructing universally optimal block designs with nested rows and columns for number of treatments greater than the number of columns. By allowing a near maximum trace in $\Delta_{v, p . q}$, we propose an initial row-column design to achieve a completely symmetric information matrix in much lesser than $v$ ! blocks. This constructive method is then extended to the case when balanced incomplete block design is given in the columns.
\end{abstract}

\section{Introduction}

A block design with nested rows and columns (BNRC) is a design which has $v$ treatments and $b$ blocks with $p \times q$ experimental units in each. The units in each block are arranged in two directions, i.e. two nested blocking factors are used; one nested blocking factor consists of $p$ levels, and the other consists of $q$ levels. Therefore, each block is a $p \times q$ row-column design. When $b=1$, a BNRC is an ordinary row-column design. Let $\Xi_{v, p, q, b}$ be a class of BNRC designs under the specified design parameters $v, p, q$ and $b$. Let $\Delta_{v, p, q}$ be a class of row-column designs under the specified design parameters $v, p$, and $q$. The information matrix associated with treatments under a design $d \in \Delta_{u, p, q}$ is well known to be

$$
C_{d}=r_{d}^{\delta}-\frac{1}{q} N_{d_{1}} N_{d_{1}}^{t}-\frac{1}{p} N_{d_{2}} N_{d_{2}}^{t}+\frac{1}{p q} N_{d} N_{d}^{t}
$$

where $r_{d}^{\delta}$ is the $v \times v$ diagonal matrix of replication numbers of the treatments, $N_{d_{1}}$ and $N_{n_{2}}$ are the treatment-row and treatment-column incidence matrices, and $N_{d}=\left[r_{d_{i}}\right]$ is

Received July 7, 1994; revised October 8, 1994.

Key words and phrases. Balanced icomplete block design (BIBD), row-column design, nested rows and columns, universally optimal design.

Research supported by National Science Council of the Republice of China. (NSC 84-2121-M009013) 
the $v \times 1$ matrix, where $r_{d_{i}}$ is the number of times the $i^{\text {th }}$ treatment appears in the block. Futhermore, the matrix $C_{d}$ is symmetric with zero row sums.

Lemma 1.1. Let $d=\left[d_{1}, d_{2}, \cdots, d_{b}\right]$ be a design in $\Xi_{v, p, q, b}$. Then $C_{d}=$ $\sum_{t=1}^{b} C_{d_{t}}$, where $C_{d_{t}}$ is the information matrix for $t^{\text {th }}$ block, $d_{t}$.

A mumber of methods for constructing BNRC's have been developed. Singh and Dey(1979) and Agrawal and Prasad (1983, 1984) give some balanced incomplete BNRC's by using the method of difference. Cheng (1986) constructs some balanced incomplete BNRC's by combining balanced incomplete block designs and balanced BNRC's. Chang and Notz $(1989,1990)$ construct universally optimal BNRC. Kiefer's (1975) definition of universal optimality and the sufficient condition for achieving universal optimality are stated below.

Definition. Let $B_{v, 0}$ be the class of $v \times v$ symmetric nonnegative definite matrices with zero row sums. Then a design $d^{*} \in \Delta_{v, p, q}$ is called universally optimal if it minimizes $\Phi\left(C_{d}\right)$ for every $\Phi: B_{v, 0} \rightarrow(-\infty, \infty]$ satisfying the following conditions:

(a) $\Phi$ is convex;

(b) for any $C_{d} \in B_{v, 0}, \Phi\left(\lambda C_{d}\right)$ is nonincreasing in the scalar $\lambda \geq 0$; and

(c) $\Phi$ is invariant under each permutation of rows and columns of $C_{d}$ in $B_{u, 0}$.

Theorem 1.2. Let $C=\left\{C_{d}: d \in \Delta_{v, p, q}\right\}$ be a class of matrices in $B_{v, 0}$. Suppose $\exists d^{*} \in \Delta_{v, p, q}$, such that $C_{d^{*}}$ satisfies

(a) $C_{d^{*}}$ is completely symmetric. (That is, $C_{d^{*}}=a I_{v}+b J_{v}$, where $a, b$ are scalars,

$I_{v}$ is the $v \times v$ identity matrix, and $J_{v}$ is the $v \times v$ matrix consisting of all 1's.)

(b) $\operatorname{tr} C_{d^{*}}=\max \operatorname{tr} C_{d}$, over all $d \in \Delta_{v, p, q}$.

Then $d^{*}$ is universally optimal in the class $\Delta_{v, p, q}$.

Using Lemma 1.1 and Theorem 1.2, Chang and Notz(1989) find universally optimal BNRC's in the lemma below.

Lemma 1.3. Let $\hat{d}$ be a row-column design in $\Delta_{v, p, q}$ such that $\operatorname{tr} C_{\hat{d}}=$ $\max _{d \in \Delta} \operatorname{tr} C_{d}$. Permute the treatment labels and let $d^{*}=\left\{\hat{d}_{1}, \hat{d}_{2}, \cdots \hat{d}_{v !}\right\}$, i.e. $d^{*}$ is the BNRC with the $t$-th block being the row-column design $\hat{d}_{t}$ where $\hat{d}_{t} \in \Delta_{v, p, q}$ is the design resulting from $\hat{d}$ by one of the $v$ ! permutations of the $v$ treatment labels. Then $d^{*}$ is a universally optimal BNRC in the class $\Xi_{v, p, q, v !}$.

A sufficient condition, given by Chang and Notz(1989), for an initial design having maximum trace and as a basis for an optimal BNRC by Lemma 1.3 is stated in the following.

Theorem 1.4. Given $v, p, q(p \leq q)$, and $v \nmid p$, let $\hat{d}$ be a design in $\Delta_{v, p, q}$ which satisfies

(a) $\left|n_{\hat{d} i j}(R)-\frac{q}{v}\right|<1,\left|n_{\hat{d} i j}(C)-\frac{p}{v}\right|<1, \forall i, j$, 
i.e. the $n_{\hat{d} i j}(K)$ are as nearly equal as possible, $K=R$ or $C$.

(b) $r_{i}= \begin{cases}p\left(I_{1}+1\right), & i=1,2, \ldots, \rho_{1} ; \text { and } \\ p I_{1}, & i=\rho_{1}+1 \ldots, v,\end{cases}$

where $r_{i}=$ the number of replications of treatment $i, I_{1}=\operatorname{int}\left(\frac{q}{v}\right)$, and

$\rho_{1}=\left(\right.$ fraction part of $\left.\frac{q}{v}\right) \times v$. Then $\operatorname{tr} C_{\hat{d}}=\max _{d \in \Delta} \operatorname{tr} C_{d}$.

Note that, this initial design contains at most $q$ varied treatments. So when $v>q$, the method stated in Theorem 1.4 is not desirable.

In section 2 , we propose an initial design in $\Delta_{v, p, q}$ contains $v$ treatments with $v>$ $q \geq p$ and $p q \geq v$, which has maximum trace. An universally optimal BNRC can be constructed by using a simple permutation method. Examples are shown in the end of this section.

In many instances, maximum trace and completely symmetry from the information matrix of a design cannot be achieved simultaneously. If an initial design has maximum trace, the BNRC may need $v$ ! blocks to achieve completely symmetry. In section 3 , we present a simple method for constructing initial design. The design has nearly maximum trace when number of columns is large, but it does not need $v$ ! blocks to achieve completely symmetry.

\section{The Construction of an Initial Design with $v>q$}

From information matrix $C_{d}$ of Eq.(1) for a row-column design, we have

$$
t r C_{d}=p q-\frac{1}{q} \sum_{i=1}^{v} \sum_{k=1}^{p} n_{d i k}^{2}(R)-\frac{1}{p} \sum_{i=1}^{v} \sum_{h=1}^{q} n_{d i h}^{2}(C)+\frac{1}{p q} \sum_{i=1}^{v} r_{d_{i}}^{2}
$$

where

$n_{d i k}(R)=$ the number of times, treatment $i$ occurs in the $k^{\text {th }}$ row,

$n_{d i h}(C)=$ the number of times, treatment $i$ occurs in the $h^{\text {th }}$ column, and

$r_{d_{i}}=$ the number of replications of treatment $i$.

In order to maximize $\operatorname{tr} C_{d}$, we need to minimize $\sum \sum n_{d i a}^{2}(K), a=k$ or $h$, with constraint $\sum \sum n_{d i a}(K)=p q$, for $K=R$ or $C$, and maximize $\sum_{i=1}^{v} r_{d_{i}}^{2}$ with constraint $\sum_{i=1}^{v} r_{d_{i}}=$ $p q$. A sufficient condition for a design in $\Delta_{v, p, q}$ to minimize $\sum_{i=1}^{v} \sum_{k=1}^{p} n_{d i k}^{2}(R)$ and $\sum_{i=1}^{v} \sum_{h=1}^{q} n_{d i h}^{2}(C)$ is stated in the following.

Theorem 2.1. Given $v, p, q(p \leq q)$, let $\bar{d}$ be a design in $\Delta_{v, p, q}$ which satisfies

$$
\left|n_{\bar{d} i k}(R)-\frac{q}{v}\right|<1, \text { and }\left|n_{\bar{d} i h}(C)-\frac{p}{v}\right|<1, \quad \forall i, k, h .
$$


Then

$$
\begin{aligned}
& \sum_{i=1}^{v} \sum_{k=1}^{p} n_{\overline{d i k}}^{2}(R)=\min _{d \in \Delta} \sum_{i=1}^{v} \sum_{k=1}^{p} n_{d i k}^{2}(R)=p\left[\left(v-\gamma_{2}\right) l_{2}^{2}+\gamma_{2}\left(l_{2}+1\right)^{2}\right], \text { and } \\
& \sum_{i=1}^{v} \sum_{h=1}^{q} n_{\bar{d} i k}^{2}(C)=\min _{d \in \Delta} \sum_{i=1}^{v} \sum_{h=1}^{q} n_{d i k}^{2}(C)=q\left[\left(v-\gamma_{1}\right) l_{1}^{2}+\gamma_{1}\left(l_{1}+1\right)^{2}\right]
\end{aligned}
$$

where $l_{1}=\operatorname{int}\left(\frac{p}{v}\right), \gamma_{1}=\left(\right.$ fraction part of $\left.\frac{p}{v}\right) v, l_{2}=\operatorname{int}\left(\frac{q}{v}\right)$, and $\gamma_{2}=\left(\right.$ fraction part of $\left.\frac{q}{v}\right) v$.

Proof. According to the method of Lagrange multiplier, we consider

$$
\begin{aligned}
& F_{1}=\sum_{i=1}^{v} \sum_{k=1}^{p} n_{d i k}^{2}(R)+\lambda_{1}\left(\sum_{i=1}^{v} \sum_{k=1}^{p} n_{d i k}(R)-p q\right), \text { and } \\
& F_{2}=\sum_{i=1}^{v} \sum_{h=1}^{p} n_{d i h}^{2}(C)+\lambda_{2}\left(\sum_{i=1}^{v} \sum_{h=1}^{p} n_{d i h}(C)-p q\right) .
\end{aligned}
$$

Then

$$
\begin{aligned}
& \frac{\partial F_{1}}{\partial n_{d i k}(R)}=2 n_{d i k}(R)+\lambda_{1}=0, \quad \forall i, k, \text { and } \\
& \frac{\partial F_{2}}{\partial n_{d i h}(C)}=2 n_{d i h}(C)+\lambda_{2}=0, \quad \forall i, h .
\end{aligned}
$$

Solving these equations, we have

$$
\begin{aligned}
& n_{d i 1}(R)=n_{d i 2}(R)=\cdots=n_{d i p}(R), i=1, \cdots, v, \sum_{i=1}^{v} \sum_{k=1}^{p} n_{d i k}(R)=p q, \text { and } \\
& n_{d i 1}(C)=n_{d i 2}(C)=\cdots=n_{d i q}(C), i=1, \cdots, v, \sum_{i=1}^{v} \sum_{h=1}^{p} n_{d i h}(C)=p q .
\end{aligned}
$$

In other words, $n_{d i k}(R)=\operatorname{int}\left(\frac{q}{v}\right)$ or $\operatorname{int}\left(\frac{q}{v}\right)+1$, and $n_{d i h}(C)=\operatorname{int}\left(\frac{p}{v}\right)$ or $\operatorname{int}\left(\frac{p}{v}\right)+1$ (i.e. $\left|n_{d i k}(R)-\frac{q}{v}\right|<1$ and $\left.\left|n_{d i h}(C)-\frac{p}{v}\right|<1, \forall i, k, h\right)$. From this results, $\sum \sum n_{d i k}^{2}(R)$ and $\sum \sum n_{d i h}^{2}(C)$ can be minimized for $d \in \Delta$. From (3), we can conclude that

$$
\begin{aligned}
& \sum_{i=1}^{v} \sum_{k=1}^{p} n_{\overline{d i k}}^{2}(R)=\min _{d \in \Delta} \sum_{i=1}^{v} \sum_{k=1}^{p} n_{d i k}^{2}(R), \text { and } \\
& \sum_{i=1}^{v} \sum_{h=1}^{q} n_{\bar{d} i h}^{2}(C)=\min _{d \in \Delta} \sum_{i=1}^{v} \sum_{h=1}^{q} n_{d i h}^{2}(C) .
\end{aligned}
$$

For design $\bar{d}$, in each row, we have $\left(v-\gamma_{2}\right)$ treatments occured $l_{2}$ times and $\gamma_{2}$ treatments occured $l_{2}+1$ tinies, in each column, we have $\left(v-\gamma_{1}\right)$ treatments occured $l_{1}$ times and 
$\gamma_{1}$ treatments occured $l_{1}+1$ times. This implies

$$
\begin{aligned}
& \sum_{i=1}^{v} \sum_{k=1}^{p} n_{\bar{d} i k}^{2}(R)=p\left[\left(v-\gamma_{2}\right) l_{2}^{2}+\gamma_{2}\left(l_{2}+1\right)^{2}\right], \text { and } \\
& \sum_{i=1}^{v} \sum_{h=1}^{q} n_{\bar{d} i h}^{2}(C)=q\left[\left(v-\gamma_{1}\right) l_{1}^{2}+\gamma_{1}\left(l_{1}+1\right)^{2}\right] .
\end{aligned}
$$

This concludes the proof.

In many instance, treatments number is greater than columns number. In case that each treatment occurs at least once in every block, a sufficient condition for an initial design to have maximun trace is stated in the following.

Theorem 2.2. Let $\check{\Delta}_{v, p, q}$ be a subclass of $\Delta_{v, p, q}$ with parameters $v>q \geq p$, $p q \geq v$ and $r_{\check{d} i} \geq 1$ (i.e. each treatment occurs at least once). Let $\check{d} \in \check{\Delta}_{v, p, q}$ which satisfies

$$
\begin{aligned}
& \text { (a) } n_{\check{d} i k}(R)=0 \text { or } 1 \text { and } n_{\check{d} i h}(C)=0 \text { or } 1 ; \forall i, k, h \text {, and } \\
& \text { (b) } r_{\check{d} i}= \begin{cases}p_{1}, & i=1,2, \ldots, c_{1} ; \\
p_{1}-1, & i=c_{1}+1, \ldots, q ; \text { and } \\
1, & i=q+1, \ldots, v,\end{cases}
\end{aligned}
$$

where $r_{\check{d} i}$ is the number of replications of treatment $i$ in $\check{d}, c_{1}=q-($ fraction part of $\left.\frac{v-q}{q}\right) q$, and $p_{1}=p-i n t\left(\frac{v-q}{q}\right)$. Then $\operatorname{tr} C_{\check{d}}=\max x_{d \in \check{\Delta}} \operatorname{tr} C_{d}$.

Proof. Since condition (a) satisfies (3), this implies that $\sum \sum n_{\tilde{d} i k}^{2}(R)=\min _{d \in \check{\Delta}}$ $\sum \sum n_{d i k}^{2}(R)=p q$ and $\sum \sum n_{\tilde{d} i h}^{2}(C)=\min _{d \in \check{\Delta}} \sum \sum n_{\tilde{d} i h}^{2}(C)=p q$. Thus one only needs to show $\sum_{i=1}^{v} r_{\tilde{d} i}^{2}$ being maximum with $\breve{d} \in \check{\Delta}$.

Design $\hat{d}$, as described in Theorem 1.4, has maximum trace in $\Delta_{v, p, q}$ with $r_{\hat{d} 1}=$ $r_{\hat{d} 2}=\cdots=r_{\hat{d} q}=p$, and $r_{\hat{d}(q+1)}=r_{\hat{d}(q+2)}=\cdots=r_{\hat{d} v}=0$ (when $v>q$ ). Then $\sum_{i=1}^{v} r_{\hat{d} i}^{2}=q p^{2}=\max _{d \in \Delta} \sum_{i=1}^{v} r_{d_{i}}^{2}$. Since it also satisfies (3), so $\sum \sum n_{\hat{d} i k}^{2}(R)=p q$ and $\sum \sum n_{\hat{d}_{i h}}^{2}(C)=p q$.

We now show that when $r_{\hat{d} j}$ increases for $j=q+1, q+2, \cdots, v$, the maximum value $\sum_{i=1}^{v} r_{\hat{d} i}^{2}$ decreases (subject to $\sum_{i=1}^{v} r_{\hat{d} i}=p q$ ). Let $\hat{d}^{\prime}$ be a design satisfying condition (a) with $r_{\hat{d}^{\prime}(q+1)}=m_{1},\left(0<m_{1}<\frac{p}{2}\right.$, and $\left.m_{1}=m_{11}+m_{12}+\cdots+m_{1 q}\right)$, and $r_{\hat{d}^{\prime}(q+2)}=$ $\cdots=r_{\hat{d}^{\prime}(v)}=0$. Then $r_{\hat{d}^{\prime} 1}+r_{\hat{d}^{\prime} 2}+\cdots+r_{\hat{d}^{\prime} q}=p q-m_{1}$. The difference between $\sum_{i=1}^{v} r_{\hat{d} i}^{2}$ and $\sum_{i=1}^{v} r_{\hat{d}^{\prime} i}^{2}$ is in the following:

$$
\begin{aligned}
R & =\sum_{i=1}^{v} r_{\hat{d}^{2} i}^{2}-\sum_{i=1}^{v} r_{\hat{d}^{\prime} i}^{2}=q p^{2}-\sum_{i=1}^{v} r_{\hat{d}^{\prime} i}^{2} \\
& =-m_{1}^{2}+\left[p^{2}-\left(p-m_{11}\right)^{2}\right]+\cdots+\left[p^{2}-\left(p-\left(m_{1}-m_{11}-\cdots-m_{1(q-1)}\right)\right)^{2}\right]
\end{aligned}
$$

Then $\frac{\partial R}{\partial m_{1}}=2\left(p-2 m_{1}\right)>0$. This implies that $\sum r_{\hat{d} i}^{2}$ decreases (subject to $\sum_{i=1}^{v} r_{\hat{d} i}=$ $p q)$ when $m_{1}$ increases. Therefore $\sum r_{\hat{d}^{\prime} i}^{2}$ has the largest value when $r_{\hat{d}^{\prime}(q+1)}=m_{1}=$ 
1. Extending this result, we conclude that $\sum_{i=1}^{v} r_{\breve{d} i}^{2}$ is maximum if we put $r_{\breve{d}(q+1)}=$ $r_{\check{d}(q+2)}=\cdots=r_{\check{d} v}=1$, in case that each treatment occurs at least onec. So $\operatorname{tr} C_{\check{d}}=$ $\max _{d \in \check{\Delta}} \operatorname{tr} C_{d}$.

The resulting design $\check{d}$ looks like the following:

$$
\left[\begin{array}{cccccccc}
q+1 & q+2 & \cdots & \cdots & \ldots & \cdots & \cdots & q+q \\
q+(q+1) & \ldots & \cdots & v & 1 & 2 & \cdots & c_{1} \\
1 & 2 & \cdots & \cdots & \ldots & \cdots & \cdots & q \\
q & 1 & 2 & \cdots & \ldots & \cdots & \cdots & q-1 \\
\vdots & \vdots & & & & & & \vdots \\
q-p_{1}+3 & q-p_{1}+4 & \cdots & \ldots & 1 & \ldots & \cdots & q-p_{1}+2
\end{array}\right]
$$

Note that, if $q \mid v, r_{\breve{d} i}$ is represented in the following:

$$
r_{\tilde{d} i}= \begin{cases}p-\frac{v-q}{q} & i=1,2, \ldots, q \\ 1 & i=q+1, \ldots, v .\end{cases}
$$

We use some examples to show that the design $\check{d}$ with the above structure exists.

Example 1. Consider a design with parameters $v=11, p=4$, and $q=4$, where $v \not p$. By the above theorem, we have $c_{1}=1$ and $p_{1}=3$. The following initial designs $\check{d}_{1}$ and $\hat{d}_{1}$ satisfy respectively the conditions listed in Theorem 2.2 and Theorem 1.4.

\begin{tabular}{lllllllll}
\multicolumn{4}{c}{$\operatorname{BLOCK}\left(\tilde{d}_{1}\right)$} & \multicolumn{4}{c}{$\operatorname{BLOCK}\left(\hat{d}_{1}\right)$} \\
05 & 06 & 07 & 08 & 01 & 02 & 03 & 04 \\
09 & 10 & 11 & 01 & 04 & 01 & 02 & 03 \\
01 & 02 & 03 & 04 & 03 & 04 & 01 & 02 \\
04 & 01 & 02 & 03 & 02 & 03 & 04 & 01
\end{tabular}

Example 2. Consider a design with parameters $v=10, p=4$, and $q=5$, where $q \mid v$. The following initial designs $\tilde{d}_{1}$ and $\hat{d}_{1}$ satisfy respectively the conditions listed in Theorem 2.2 and Theorem 1.4.

\begin{tabular}{lllllllllll}
\multicolumn{4}{c|}{$\operatorname{BLOCK}\left(\check{d}_{1}\right)$} & \multicolumn{4}{c}{$\operatorname{BLOCK}\left(\hat{d}_{1}\right)$} \\
6 & 7 & 8 & 9 & 0 & 1 & 2 & 3 & 4 & 5 \\
1 & 2 & 3 & 4 & 5 & 2 & 3 & 4 & 5 & 1 \\
2 & 3 & 4 & 5 & 1 & 3 & 4 & 5 & 1 & 2 \\
3 & 4 & 5 & 1 & 2 & 4 & 5 & 1 & 2 & 3
\end{tabular}

The following example shows that we need only 12 blocks to obtain completely symmetric information matrix. (The result mentioned in Theorem 1.4 needs 24 blocks.) 
Example 3. Consider a design with parameters $v=4, p=2$, and $q=3$. In the followings, there are two initial designs $\hat{d}_{1}$ and $\breve{d}_{1}$, where $\breve{d}_{1}$ satisfies the conditions listed in Theorem 2.2, and $\hat{d}_{1}$ satisfies those of Theorem 1.4.



Take elevem permutations for the treatment labels in design $\check{d}_{1}$, i.e.

$$
\{(43),(23),(324),(341),(1234),(412),(23)(14),(2314),(24),(342),(3124)\}
$$

we obtain $\check{d}_{2}, \check{d}_{3}, \cdots, \check{d}_{12}$ in the following:

\section{BLOCK}

$\begin{array}{llllllllll}312 & 413 & 314 & 132 & 123 & 124 \\ 124 & 132 & 142 & 324 & 234 & 243\end{array}$

$\begin{array}{llllllll}143 & 243 & 214 & 213 & 3 & 24 \\ 432 & 431 & 143 & 134 & 24\end{array}$

Let $d^{*}=\left\{\check{d}_{1}, \check{d}_{2}, \cdots, \check{d}_{12}\right\}$, it is an universally optimal BNRC in $\Xi_{4,2,3,12}$.

\section{Construct an Initial Design with Trace Nearly Maximum}

The method of the construction is described in the following theorem.

Theorem 3.1. Given $v, p, q(p \leq q)$, Let $\tilde{d}$ be a design in $\Delta_{v, p, q}$ which satisfies

$$
\begin{aligned}
& \text { (a) }\left|n_{\tilde{d} i j}(R)-\frac{q}{v}\right|<1,\left|n_{\tilde{d} i j}(C)-\frac{p}{v}\right|<1, \forall i, j, \text { and } \\
& (b)\left|r_{\tilde{d} i}-\frac{p q}{v}\right|<1
\end{aligned}
$$

where $r_{\tilde{d}_{i}}$ is the number of replications of treatment $i$ in $\tilde{d}$.

Then $\operatorname{tr} C_{\tilde{d}} \rightarrow q \rightarrow \infty \max _{d \in \Delta} \operatorname{tr} C_{d}$.

Proof. If (a) is satisfied, we see that $\sum \sum n_{\tilde{d} i k}^{2}(R)$ and $\sum \sum n_{\tilde{d} i h}^{2}(C)$ will be minimized. By Theorem 1.4, we have $\sum_{i=1}^{v} r_{\hat{d} i}^{2}=\max _{d \in \Delta} \sum_{i=1}^{v} r_{d_{i}}^{2}$. So the difference 
between $\operatorname{tr} C_{\hat{d}}$ and $\operatorname{tr} C_{\tilde{d}}$ is in the following.

$$
\begin{aligned}
& \operatorname{tr} C_{\hat{d}}-\operatorname{tr} C_{\tilde{d}}=\frac{1}{p q}\left(\sum_{i=1}^{v} r_{\hat{d} i}^{2}-\sum_{i=1}^{v} r_{\tilde{d} i}^{2}\right) \\
= & \frac{1}{p q}\left[\left(v-\rho_{1}\right) p^{2} I_{1}^{2}+\rho_{1} p^{2}\left(I_{1}+1\right)^{2}\right]-\left[\left(v-\rho_{2}\right) I_{2}^{2}+\rho_{2}\left(I_{2}+1\right)^{2}\right] \\
= & \frac{1}{p q}\left(p^{2}\left[I_{1}^{2} v+\left(1+2 I_{1}\right) \rho_{1}\right]-\left(v I_{2}^{2}+2 I_{2} \rho_{2}+\rho_{2}\right)\right) \\
= & \frac{1}{p q}\left(p^{2}\left[\left(\frac{q-\rho_{1}}{v}\right)^{2} v+\left(1+2 \frac{q-\rho_{1}}{v}\right) \rho_{1}\right]-v\left(\frac{p q-\rho_{2}}{v}\right)^{2}-2 \frac{p q-\rho_{2}}{v} \rho_{2}-\rho_{2}\right) \\
= & \frac{1}{p q} \frac{p^{2} \rho_{1}\left(v-\rho_{1}\right)+\rho_{2}\left(\rho_{2}-v\right)}{v} \\
\leq & \frac{1}{p q} \frac{p^{2} v^{2}}{4 v}=\frac{p v}{4 q}
\end{aligned}
$$

where $I_{1}=\operatorname{int}\left(\frac{q}{v}\right), \rho_{1}=\left(\right.$ fraction part of $\left.\frac{q}{v}\right) \times v, I_{2}=\operatorname{int}\left(\frac{p q}{v}\right)$ and $\rho_{2}=($ fraction part of $\left.\frac{p q}{v}\right) \times v$. Since $p$ and $v$ are fixed, hence $\operatorname{tr} C_{\bar{d}} \rightarrow q \rightarrow \infty \max _{d \in \Delta} \operatorname{tr} C_{d}$.

For BNRC, if an initial row-column design $\hat{d}$, is also a BIBD when columns in $\hat{d}$ are considered as blocks, satisfies condition $(a)$ and $(b)$ in Theorem 3.1. Then we need only few blocks to obtain completely symmetric information matrix. The following example is given to show the above result.

Example 4. Consider the case of $v=6, p=3$, and $q=10$, a possible initial design $\tilde{d}_{1}$, is a $\operatorname{BIBD}(6,10,5,3,2)$ when columns in $\tilde{d}_{1}$ are considered as blocks, satisfies the conditions listed in Theorem 3.1 is presented in the following. $\hat{d}_{1}$ in the following, is a possible design in $\Delta_{6,3,10}$ satisfying Theorem 1.4 .

$$
\operatorname{BLOCK}\left(\hat{d}_{1}\right)
$$

1156635234

22115645543

3434122656
$\operatorname{BLOCK}\left(\tilde{d}_{1}\right)$

1234561234

234556122341

3456123412

The differences between $\operatorname{tr} C_{\hat{d}_{1}}$ and $\operatorname{tr} C_{\bar{d}_{1}}$ are computed.

$$
\begin{aligned}
& \operatorname{tr} C_{\hat{d}_{1}}-\operatorname{tr} C_{\tilde{d}_{1}}=20-19.6=0.4 \text { and } \\
& \frac{\operatorname{tr} C_{\hat{d}_{1}}-\operatorname{tr} C_{\tilde{d}_{1}}}{\operatorname{tr} C_{\hat{d}_{1}}}=\frac{0.4}{20}=0.02
\end{aligned}
$$

Take nine permutations for the treatment labels in $\tilde{d}_{1}$, i.e.

$$
\{(546),(456),(45),(53)(64),(523),(562),(526),(34),(53)\},
$$


we obtain $\tilde{d}_{2}, \tilde{d}_{3}, \ldots, \tilde{d}_{10}$ in the following:

\section{BLOCK}

\begin{tabular}{|c|c|c|}
\hline 1145534236 & 1164436235 & 1146634235 \\
\hline 2211456463 & 2211645653 & 2211465453 \\
\hline 636122545 & 35351222464 & 353551226646 \\
\hline 1134453256 & 1136652354 & 1162236534 \\
\hline 211346365 & 3311264245 & 55511162246643 \\
\hline 656122434 & 5454133626 & 3434155262 \\
\hline 1125 & 1156645243 & 1136653254 \\
\hline 54243 & 2211563534 & 2211364345 \\
\hline 4166525 & 4343122656 & 5454122636 \\
\hline
\end{tabular}

Let $d^{*}=\left\{\tilde{d}_{1}, \tilde{d}_{2}, \ldots, \tilde{d}_{10}\right\}$, it is a nearly universally optimal BNRC in $\Xi_{6,3,10,10}$. The method stated in Theorem 1.4 may need 6 ! blocks for completely symmetric from information matrix.

Conclusion Remark. In this paper, we propose two initial designs stated in Theorem 2.2 and Theorem 3.1 as bases for universally opotimal BNRCs. when the number of treatments $(v)$ is greater than the number of columns $(q)$, and for each treatment occured at least once in a block, then the design $\check{d}$ stated in Theorem 2.1 will have maximum trace. Furthermore, by using simple permutation method, a complete symmetric information matrix can be achieved in lesser than $v$ ! blocks. According to Theorem 3.1, if the design need larger number of columns, using $\tilde{d}$ with BIBD in its columns, we obtain an initial design with nearly maximum trace, and a nearly universally optimal BNRC can be achieved in much less than $v$ ! blocks.

\section{Reference}

[1] H. L. Agrawal and J. Prasad, "On construction of balanced incomplete block designs with nested rows and columns," Sankhya: The Indian Journal of Statistics, Series B, 45(3), 1983, 345-350.

[2] H. L. Agrawal and J. Prasad, "Construction of partially balanced incomplete block designs with nested rows and columns," Biometrical J., 26(8) 1984, 883-891.

[3] Yueh-Jane Chang and W. I. Notz, Optimal block designs with nested rows and columns, Ph. D Dissertation, Department of Statistics, The Ohio State University, 1989.

[4] Yueh-Jane Chang and W. I. Notz, "A method for constructing universally optimal block designs with nested rows and columns," Utilitas Mathematica. A Canadian J. of Applied Mathematics, Computer Science and Statistics, 38, 1990, 263-276.

[5] Ching-Shui Cheng, "A method for constructing balanced incomplete-block designs with nested rows and columns," Biometrika, 73(3), 1986, 695-700.

[6] J. Kiefer, "Construction and optimality of generalized youden designs," A Survey of Statistician Design and Linear Models, ed. J. Srivastava, New York: Norther Holland, 1975, 333-353.

[7] M. Singh and A. Dey, "Block designs with nested rows and columns," Biometrika, 66(2), 1979, 321-326. 
[8] A. P. Street, Combinatorics of Experimental Design, Oxford New York Toronto, 1987.

Department of Applied Mathematics, National Chiao Tung University, Hsinchu, Taiwan, R.O.C.

Department of Management Science, National Chiao Tung University, Hsinchu, Taiwan, R.O.C. 